CUBO A Mathematical Journal

Vol.17, $N^{-}$01, (99-106). March 2015

\title{
Semi Open sets in bispaces
}

\author{
Amar Kumar BanerJee \\ Department of Mathematics, \\ Burdwan University, \\ Burdwan-713104, W.B., India. \\ akbanerjee1971@gmail. com
}

\author{
Pratap Kumar Saha \\ Behala College, \\ Kolkata, W.B., India. \\ pratapsahaz@gmail.com
}

\begin{abstract}
The notions of semi open sets in a topological space were introduced by N.Levine in 1963. Here we study the same using the idea of $\tau_{1}\left(\tau_{2}\right)$ semi open sets with respect to $\tau_{2}\left(\tau_{1}\right)$, pairwise semi open sets in a more general structure of a bispace and investigate how far several results as valid in a bitopological space are affected in bispaces.
\end{abstract}

\section{RESUMEN}

Las nociones de conjuntos semiabiertos en un espacio topológico se introdujeron por N. Levine en 1963. Aquí estudiamos lo mismo usando la idea de conjuntos semiabiertos $\tau_{1}\left(\tau_{2}\right)$ respecto de conjuntos abiertos semiabiertos dos a dos $\tau_{2}\left(\tau_{1}\right)$, en una estructura más general de biespacio e investigamos cómo varios resultados válidos en un espacio bitopológico cambian en biespacios.

Keywords and Phrases: bispaces, semi open sets, $\tau_{1}$ semi open sets with respect to $\tau_{2}$.

2010 AMS Mathematics Subject Classification: 54A05, 54E55, 54E99 


\section{Introduction}

The notion of a $\sigma$ space or simply a space was introduced by A.D.Alexandroff [1] in 1940 generalising the idea of a topological space where only countable unions of open sets were taken to be open. In 2001 the idea of space was used by Lahiri and Das [8] to generalise the notion of a bitopological space to a bispace. N.Levine [9] introduced the concept of semi open sets in a topological space in 1963 and this idea was generalised by S.Bose [3] in the setting of a bitopological space $(\mathrm{X}, \mathcal{P}, \mathcal{Q})$ using the idea of $\mathcal{P}(\mathcal{Q})$ semi open sets with respect to $\mathcal{Q}(\mathcal{P})$ etc. Later the same was studied in a space by Lahiri and Das [7] and they critically took the matter of generalisation in this setting. Here we have studied the concept of $\tau_{1}\left(\tau_{2}\right)$ semi open sets with respect to $\tau_{2}\left(\tau_{1}\right)$ and some other properties in the setting of a bispace and have shown with typical examples how far several results as valid in 3 are affected in bispaces. Also we have given a necessary and sufficient condition for a bispace to be a bitopological space in terms of $\tau_{1}\left(\tau_{2}\right)$ semi open sets with respect to $\tau_{2}\left(\tau_{1}\right)$.

\section{Preliminaries}

Definition 2.1. [1] $A$ set $\mathrm{X}$ is called an Alexandroff space or simply a space if in it is chosen a system of subsets $\mathcal{F}$ satisfying the following axioms:

(1) The intersection of a countable number of sets from $\mathcal{F}$ is a set in $\mathcal{F}$.

(2) The union of a finite number of sets from $\mathcal{F}$ is a set in $\mathcal{F}$.

(3) The void set $\phi$ is a set in $\mathcal{F}$.

(4) The whole set $\mathrm{X}$ is a set in $\mathcal{F}$.

Sets of $\mathcal{F}$ are called closed sets. Their complementary sets are called open sets. It is clear that instead of closed set in the definition of the space, one may put open sets with subject to the condition of countable summability, finite intersectibility and the condition that $X$ and $\phi$ should be open. The collection of all such open sets will sometimes be denoted by $\tau$ and the space by $(X, \tau)$. Note that, in general $\tau$ is not a topology as can be easily seen by taking $X=R$, the set of real numbers and $\tau$ as the collection of all $F_{\sigma}$ sets in $R$.

Definition 2.2. [1] To every set $M$ of $(X, \tau)$ we correlate its closure $\bar{M}=$ the intersection of all closed sets containing M. Sometimes the closure of a set $\mathrm{M}$ will be denoted by $\tau$ clM or simply clM when there is no confusion about $\tau$.

Generally the closure of a set in a space is not a closed set.

From the axioms, it easily follows that
1) $\overline{\mathrm{M} \cup N}=\overline{\mathrm{M}} \cup \overline{\mathrm{N}}$;
2) $M \subset \bar{M}$;
3) $\bar{M}=\overline{\bar{M}}$;
4) $\bar{\phi}=\phi$. 
Definition 2.3. 77 The interior of a set $M$ in $(X, \tau)$ is defined as the union of all open sets contained in $\mathrm{M}$ and is denoted by intM or int $\mathrm{M}$ when there is no confusion.

Definition 2.4. [6] A non empty set $\mathrm{X}$ on which are defined two arbitrary topologies $\mathcal{P}, \mathcal{Q}$ is called a bitopological space and denoted by $(\mathrm{X}, \mathcal{P}, \mathcal{Q})$.

Definition 2.5. [8] Let $\mathrm{X}$ be a nonempty set. If $\tau_{1}$ and $\tau_{2}$ be two collections of subsets of $\mathrm{X}$ such that $\left(\mathrm{X}, \tau_{1}\right)$ and $\left(\mathrm{X}, \tau_{2}\right)$ are two spaces, then $\mathrm{X}$ is called a bispace and is denoted by $\left(\mathrm{X}, \tau_{1}, \tau_{2}\right)$.

\section{Pairwise Semi Open sets}

Definition 3.1. (cf. Definition 1[3] ): Let $\left(\mathrm{X}, \tau_{1}, \tau_{2}\right)$ be a bispace. We say that a subset $\mathrm{A}$ of $\mathrm{X}$ is $\tau_{1}$ semi open with respect to $\tau_{2}$ (in short $\tau_{1}$ s.o.w.r.to $\tau_{2}$ ) if and only if there exists a $\tau_{1}$ open set $\mathrm{O}$ such that $\mathrm{O} \subset \mathrm{A} \subset \tau_{2}$ clO.

Similarly $A \subset X$, is $\tau_{2}$ semi open with respect to $\tau_{1}$ (in short $\tau_{2}$ s.o.w.r.to $\tau_{1}$ ) if and only if there exists a $\tau_{2}$ open set $\mathrm{O}$ such that $\mathrm{O} \subset \mathrm{A} \subset \tau_{1} \mathrm{clO}$.

We say that $A$ is pairwise semi open if and only if it is both $\tau_{1}$ s.o.w.r.to $\tau_{2}$ and $\tau_{2}$ s.o.w.r.to $\tau_{1}$. Note that a $\tau_{1}\left(\tau_{2}\right)$ open set is $\tau_{1}\left(\tau_{2}\right)$ s.o.w.r.to $\tau_{2}\left(\tau_{1}\right)$.

Throughout our discussion, $\left(X, \tau_{1}, \tau_{2}\right)$ or simply $X$ stands for a bispace, $R$ stands for the set of real numbers, $Q$ for the set of rational numbers and $N$ stands for the set of natural numbers and sets are always subsets of $\mathrm{X}$ unless otherwise stated.

Theorem 3.2. Let $\left(\mathrm{X}, \tau_{1}, \tau_{2}\right)$ be a bispace. Let $\mathrm{A} \subset \mathrm{X}$, and $\mathrm{A}$ is $\tau_{1}$ s.o.w.r.to $\tau_{2}$ then $\tau_{2} \mathrm{cl} \mathrm{A}=$ $\tau_{2} \operatorname{cl}\left(\tau_{1}\right.$ int $\left.A\right)$.

Proof. Let $A$ is $\tau_{1}$ s.o.w.r.to $\tau_{2}$ then there exists a $\tau_{1}$ open set $O$ such that $\mathrm{O} \subset A \subset \tau_{2}$ clO. Also $\mathrm{O} \subset \tau_{1}$ int $A$. Therefore, $A \subset \tau_{2} \operatorname{clO} \subset \tau_{2} \operatorname{cl}\left(\tau_{1}\right.$ int $\left.A\right)$ and hence $\tau_{2} \operatorname{cl} A \subset \tau_{2} \operatorname{cl}\left(\tau_{2} \operatorname{cl}\left(\tau_{1}\right.\right.$ int $\left.\left.A\right)\right)=$ $\tau_{2} \operatorname{cl}\left(\tau_{1}\right.$ int $\left.A\right)$. Also $\tau_{2} \operatorname{cl}\left(\tau_{1}\right.$ int $\left.A\right) \subset \tau_{2} \operatorname{cl}$. Therefore, $\tau_{2} \operatorname{cl} A=\tau_{2} \operatorname{cl}\left(\tau_{1}\right.$ int $\left.A\right)$.

Corollary 3.3. If $\mathrm{A}$ is $\tau_{1}$ s.o.w.r.to $\tau_{2}$ and $\mathrm{A} \neq \phi$ then $\tau_{1}$ int $\mathrm{A} \neq \phi$.

Corollary 3.4. Let $\mathrm{A}$ is $\tau_{1}$ s.o.w.r.to $\tau_{2}$ and $\mathrm{A} \subset \mathrm{B}$ then $\mathrm{A} \subset \tau_{2} \operatorname{cl}\left(\tau_{1}\right.$ int $\left.\mathrm{B}\right)$.

If $\left(X, \tau_{1}, \tau_{2}\right)$ is a bitopological space then converse part of the theorem 3.2 also holds which is seen in [3] . But in a bispace, this may not be true as shown below:

Example 3.5. Let $\mathrm{X}=[0,2]$ and $\left\{\mathrm{G}_{i}\right\}$ be the collection of all countable subsets of irrational numbers in $[0,1]$. Let $\tau_{1}$ be the collection of all sets of the form $\mathrm{G}_{i} \cup\{\sqrt{2}\}$ together with $\mathrm{X}$ and $\phi$, and $\tau_{2}$ be the collection of all sets $\mathrm{G}_{\mathrm{i}}$ together with $\mathrm{X}$ and $\phi$. Then $\left(\mathrm{X}, \tau_{1}, \tau_{2}\right)$ is a bispace. Now consider a subset $\mathrm{A}=[0,1] \cup\{\sqrt{2}\}$ then $\tau_{2} \mathrm{clA}=\mathrm{X}$ and $\tau_{1}$ int $\mathrm{A}$ is set of all irrational numbers in $[0,1]$ together with $\sqrt{2}$ and hence $\tau_{2} \operatorname{cl}\left(\tau_{1}\right.$ int $\left.A\right)=X$. Therefore, $\tau_{2} \operatorname{cl} A=\tau_{2} \operatorname{cl}\left(\tau_{1} i n t A\right)$. But for any $\tau_{1}$ 
open set $\mathrm{G}(\neq \mathrm{X}, \phi), \tau_{2} \mathrm{clG}=\mathrm{G} \cup \mathrm{Q}_{1} \cup[1,2]$, where $\mathrm{Q}_{1}$ is the set of all rational numbers in $[0,1]$. Clearly $\tau_{2} \mathrm{clG}$ does not contain $\mathrm{A}$. Therefore, there does not exist any $\tau_{1}$ open set $\mathrm{G}$ satisfying $\mathrm{G} \subset \mathrm{A} \subset \tau_{2}$ clG. So $\mathrm{A}$ is not $\tau_{1}$ s.o.w.r.to $\tau_{2}$.

However we observe in the following theorem that the converse part of theorem 3.2 holds under an additional condition.

Theorem 3.6. In a bispace $\left(X, \tau_{1}, \tau_{2}\right)$, let $\tau_{2} \operatorname{cl} A=\tau_{2} \operatorname{cl}\left(\tau_{1}\right.$ int $\left.A\right)$. Then $A$ is $\tau_{1}$ s.o.w.r.to $\tau_{2}$ for any subset $\mathrm{A}$ of $\mathrm{X}$ if the condition $\mathrm{C}_{1}$ is satisfied.

$\mathrm{C}_{1}$ : Arbitrary union of $\tau_{1}$ open sets is $\tau_{1}$ s.o.w.r.to $\tau_{2}$.

Proof. Let $\mathrm{O}=\tau_{1}$ int $A$. Then by the condition $\mathrm{C}_{1}, \mathrm{O}$ is $\tau_{1}$ s.o.w.r.to $\tau_{2}$. So there exists a $\tau_{1}$ open set $G$ such that $G \subset O \subset \tau_{2}$ clG. Now since $\tau_{2} \operatorname{clO}=\tau_{2} \operatorname{cl}\left(\tau_{1}\right.$ int $\left.A\right)=\tau_{2}$ clA and $\mathrm{O} \subset \tau_{2} \mathrm{clG}$, it follows that $\tau_{2} \mathrm{clO} \subset \tau_{2} \mathrm{cl}\left(\tau_{2} \mathrm{clG}\right)=\tau_{2} \mathrm{clG}$ and hence $\tau_{2} \mathrm{clA} \subset \tau_{2} \mathrm{clG}$. Therefore, $\mathrm{G} \subset \mathrm{O} \subset \mathrm{A} \subset \tau_{2} \mathrm{clA} \subset \tau_{2} \mathrm{clG}$ and so $A$ is $\tau_{1}$ s.o.w.r.to $\tau_{2}$.

Remark 3.7. We see in the Example 3.8 below that there is a bispace which is not a bitopological space where the condition $\mathrm{C}_{1}$ holds good.

Example 3.8. Let $\mathrm{X}=[0,2], \tau_{1}$ be the collection of all sets $\mathrm{G}_{\mathrm{i}}$ together with $\mathrm{X}$ and $\phi$ and $\tau_{2}$ be the collection of all sets $F_{i}$ together with $X$ and $\phi$ where $\left\{G_{i}\right\}$ and $\left\{F_{i}\right\}$ are the collection of all countable subsets of irrational numbers in $[0,1]$ and $[1,2]$ respectively. Then $\left(X, \tau_{1}, \tau_{2}\right)$ is a bispace but not a bitopological space. Now consider all $\tau_{1}$ open sets $\left\{\mathrm{G}_{i}\right\}$. Then $\cup \mathrm{G}_{i}$ is the set of all irrational numbers in $[0,1]$ which is not $\tau_{1}$ open. But since, for any $\tau_{1}$ open set $\mathrm{G}_{i}, \tau_{2} \mathrm{clG} \mathrm{G}_{i}=[0,1] \cup \mathrm{Q}_{2}$ where $\mathrm{Q}_{2}$ is set of all rational numbers in $[1,2]$. It follows that $\mathrm{G}_{\mathrm{i}} \subset \cup \mathrm{G}_{\mathrm{i}} \subset \tau_{2} \mathrm{cl} \mathrm{G}_{i}$. This implies that $\cup \mathrm{G}_{\mathrm{i}}$ is $\tau_{1}$ s.o.w.r.to $\tau_{2}$ although it is not $\tau_{1}$ open.

Theorem 3.9. Countable union of $\tau_{1}$ s.o.sets w.r.to $\tau_{2}$ is $\tau_{1}$ s.o.w.r.to $\tau_{2}$.

Proof. Let $\left\{A_{n}: n \in N\right\}$ be a countable collection of $\tau_{1}$ s.o.sets w.r.to $\tau_{2}$. Then for each $n \in N$ there exists a $\tau_{1}$ open set $O_{n}$ such that $O_{n} \subset A_{n} \subset \tau_{2} c l O_{n}$. This implies that $\cup\left\{O_{n}: n \in N\right\}$ $\subset \cup\left\{A_{n}: n \in N\right\} \subset \cup\left\{\tau_{2} c l O_{n}: n \in N\right\} \subset \tau_{2} c l\left(\cup\left\{O_{n}: n \in N\right\}\right)$ i.e, $O \subset \cup\left\{A_{n}: n \in N\right\} \subset \tau_{2} c l O$, where $O=\cup\left\{O_{n}: n \in N\right\}$, a $\tau_{1}$ open set. Hence $\cup\left\{A_{n}: n \in N\right\}$ is $\tau_{1}$ s.o.w.r.to $\tau_{2}$.

Remark 3.10. In [3] it was proved that in a bitopological space arbitrary union of $\tau_{1}$ s.o.sets w.r.to $\tau_{2}$ is $\tau_{1}$ s.o.w.r.to $\tau_{2}$. But this may not be true in a bispace as shown in the Example 3.11 below.

Example 3.11. Consider $X=R$. Let $\tau_{1}$ open sets are $X, \phi$ and all sets $G_{i}$ where $\left\{G_{i}\right\}$ is the collection of all countable subsets of irrational numbers in $\mathrm{R}$ and $\tau_{2}$ open sets are $\mathrm{X}, \phi$ and all $\mathrm{F}_{\sigma}$ sets in $\mathrm{R}$. Clearly $\tau_{2}$ closed sets are the $\mathrm{G}_{\delta}$ sets. So for any subset $\mathrm{G}$ in $\mathrm{R}$ we have $\tau_{2} \mathrm{clG}=\mathrm{G}$. Therefore, for any set $\mathrm{A}$ which is $\tau_{1}$ s.o.w.r.to $\tau_{2}$ in $\mathrm{X}$, there exists a $\tau_{1}$ open set $\mathrm{G}_{i}$ such that $\mathrm{G}_{i} \subset \mathrm{A} \subset \tau_{2} \mathrm{clG}_{i}=\mathrm{G}_{i}$. This implies that $\mathrm{A}=\mathrm{G}_{i}$, i.e., $\mathrm{A}$ is $\tau_{1}$ open set. So $\tau_{1}$ open sets are the only $\tau_{1}$ s.o.sets w.r.to $\tau_{2}$. Since the union of all $\tau_{1}$ open sets $\mathrm{G}_{i}\left(\mathrm{G}_{i} \neq \mathrm{X}\right)$ is precisely the set 
of all irrational numbers in $\mathrm{R}$ which is not $\tau_{1}$ open, it follows that arbitrary union of $\tau_{1}$ s.o.sets w.r.to $\tau_{2}$ may not be $\tau_{1}$ s.o.w.r.to $\tau_{2}$.

However the additional condition $\mathrm{C}_{1}$ ensures the result in theorem 3.9 for arbitrary union.

Theorem 3.12. Arbitrary union of $\tau_{1}$ s.o.sets w.r.to $\tau_{2}$ is $\tau_{1}$ s.o.w.r.to $\tau_{2}$ if and only if the condition $\mathrm{C}_{1}$ is satisfied.

Proof. Assume first that the arbitrary union of $\tau_{1}$ s.o.sets w.r.to $\tau_{2}$ is $\tau_{1}$ s.o.w.r.to $\tau_{2}$. Since every $\tau_{1}$ open set is $\tau_{1}$ s.o.w.r.to $\tau_{2}$, arbitrary union of $\tau_{1}$ open sets is $\tau_{1}$ s.o.w.r.to $\tau_{2}$, i.e., the condition $\mathrm{C}_{1}$ holds.

Next assume that the condition $C_{1}$ holds. Let $\left\{A_{i}\right\}$ be an arbitrary collection of $\tau_{1}$ s.o.sets w.r.to $\tau_{2}$ and $A=\cup A_{i}$. For each $i$, there exists a $\tau_{1}$ open set $G_{i}$ such that $G_{i} \subset A_{i} \subset \tau_{2} c l G_{i}$. Therefore, $\cup G_{i} \subset \cup A_{i}=A \subset \cup \tau_{2} c l G_{i} \subset \tau_{2} c l\left(\cup G_{i}\right)$. Since $\cup G_{i}$, by assumption, is $\tau_{1}$ s.o.w.r.to $\tau_{2}$, there exists a $\tau_{1}$ open set $G$ such that $G \subset \cup G_{i} \subset \tau_{2}$ clG. Therefore, $G \subset \cup G_{i} \subset A \subset \cup \tau_{2} c l_{i} \subset$ $\tau_{2} \operatorname{cl}\left(\cup G_{i}\right) \subset \tau_{2} c l \tau_{2} \operatorname{clg}=\tau_{2} c l G$. This proves that $A$ is $\tau_{1}$ s.o.w.r.to $\tau_{2}$.

Theorem 3.13. Let $\mathrm{A}$ be $\tau_{1}$ s.o.w.r.to $\tau_{2}$ in a bispace $\left(\mathrm{X}, \tau_{1}, \tau_{2}\right)$ and let $\mathrm{A} \subset \mathrm{B} \subset \tau_{2}$ clA. Then $\mathrm{B}$ is $\tau_{1}$ s.o.w.r.to $\tau_{2}$.

Proof. Since $A$ is $\tau_{1}$ s.o.w.r.to $\tau_{2}$, there exists a $\tau_{1}$ open set $O$ such that $O \subset A \subset \tau_{2}$ clO. Therefore, $\mathrm{O} \subset \mathrm{A} \subset \mathrm{B} \subset \tau_{2} \operatorname{cl} \mathrm{A} \subset \tau_{2} \operatorname{cl}\left(\tau_{2} \mathrm{clO}\right)=\tau_{2} \mathrm{clO}$ and hence $\mathrm{B}$ is $\tau_{1}$ s.o.w.r.to $\tau_{2}$.

Theorem 3.14. Let $\left(\mathrm{X}, \tau_{1}, \tau_{2}\right)$ be a bispace and let $\mathrm{A} \subset \mathrm{Y} \subset \mathrm{X}$. If $\mathrm{A}$ is pairwise s.o. in $\mathrm{X}$, it is pairwise s.o. in $\mathrm{Y}$.

Proof. Let $A$ be $\tau_{1}$ s.o.w.r.to $\tau_{2}$. Then there exists a $\tau_{1}$ open set $\mathrm{O}$ such that $\mathrm{O} \subset \mathrm{A} \subset \tau_{2} \mathrm{clO}$. Let $\mathrm{O}_{Y}=\mathrm{Y} \cap \mathrm{O}$ which is $\tau_{1}$ open in $\mathrm{Y}$. So $\mathrm{O}_{\mathrm{Y}}=\mathrm{Y} \cap \mathrm{O} \subset \mathrm{Y} \cap \mathrm{A} \subset \mathrm{Y} \cap \tau_{2} \mathrm{clO}=\tau_{2} \mathrm{clO}_{\mathrm{Y}}$ in $\mathrm{Y}$. Interchanging the role of $\tau_{1}$ and $\tau_{2}$ we get the result.

We denote the class of all $\tau_{1}$ s.o.w.r.to $\tau_{2}$ by $\tau_{1}$ S.O. $(X)_{\tau_{2}}$.

Theorem 3.15. Let $\mathcal{B}=\left\{\mathrm{B}_{\alpha}\right\}$ be a collection of subsets of $\mathrm{X}$ such that (i) $\tau_{1} \subset \mathcal{B}$ and (ii) $\mathrm{B} \in \mathcal{B}$ and $\mathrm{B} \subset \mathrm{D} \subset \tau_{2}$ clB imply $\mathrm{D} \in \mathcal{B}$, then $\tau_{1}$ S.O. $(\mathrm{X})_{\tau_{2}} \subset \mathcal{B}$.

Proof. Let $A \in \tau_{1}$ S.O. $(X)_{\tau_{2}}$, then there exists a $\tau_{1}$ open set $O$ such that $O \subset A \subset \tau_{2}$ clO. Therefore, $\mathrm{O} \in \mathcal{B}$ and $\mathrm{O} \subset \mathrm{A} \subset \tau_{2}$ clO imply that $A \in \mathcal{B}$ and hence the result follows.

We denote the set $\left\{\tau_{1}\right.$ int $A: A \in \tau_{1}$ S.O. $\left.(X)_{\tau_{2}}\right\}$ by $\tau_{1} \operatorname{int}\left(\tau_{1}\right.$ S.O. $\left.(X)_{\tau_{2}}\right)$. Interchanging the role of $\tau_{1}$ and $\tau_{2}$ we may denote other such classes at our will.

From the construction, it is obvious that $\tau_{1} \subset \tau_{1} \operatorname{int}\left(\tau_{1}\right.$ S.O. $\left.(X)_{\tau_{2}}\right)$. But in general, $\tau_{1}$ may not be equal to $\tau_{1} \operatorname{int}\left(\tau_{1} S . O .(X)_{\tau_{2}}\right)$ as shown in the following example. 
Example 3.16. Let $\left(\mathrm{X}, \tau_{1}, \tau_{2}\right)$ be the bispace as in Example 3.8. Now for any $\tau_{1}$ open set $\mathrm{G}_{i}$, $\tau_{2} \mathrm{clG}_{i}=[0,1] \cup \mathrm{Q}_{2}$, where $\mathrm{Q}_{2}$ is set of all rational numbers in $[1,2]$. Let $\mathrm{A}=[0,1]$. Then for any $\tau_{1}$ open set $\mathrm{G}_{i}, \mathrm{G}_{\mathrm{i}} \subset \mathrm{A} \subset[0,1] \cup \mathrm{Q}_{2}=\tau_{2} \mathrm{clG}_{i}$. This implies that $\mathrm{A}$ is $\tau_{1}$ s.o.w.r.to $\tau_{2}$, i.e., $A \in \tau_{1}$ S.O. $(X)_{\tau_{2}}$. But $\tau_{1}$ intA is the set of all irrational numbers in [0,1] which is not $\tau_{1}$ open.

However equality $\tau_{1}=\tau_{1} \operatorname{int}\left(\tau_{1}\right.$ S.O. $\left.(\mathrm{X})_{\tau_{2}}\right)$ holds if an additional condition holds.

Theorem 3.17. In a bispace $\left(X, \tau_{1}, \tau_{2}\right), \tau_{1}=\tau_{1} \operatorname{int}\left(\tau_{1}\right.$ S.O. $\left.(X)_{\tau_{2}}\right)$ if and only if the condition $\mathrm{C}_{2}$ is satisfied.

$\mathrm{C}_{2}:$ For any $\mathrm{A} \subset \mathrm{X}$ which is $\tau_{1}$ s.o.w.r.to $\tau_{2}$, there exists a maximal $\tau_{1}$ open set $\mathrm{O}$ such that $\mathrm{O} \subset \mathrm{A} \subset \tau_{2} \mathrm{clO}$.

Proof. First assume that $\tau_{1}=\tau_{1} \operatorname{int}\left(\tau_{1}\right.$ S.O. $\left.(X)_{\tau_{2}}\right)$, and let $A$ be any subset of $X$ which is $\tau_{1}$ s.o.w.r.to $\tau_{2}$. Then $\tau_{1}$ int $A \in \tau_{1}$. Also by theorem 3.2, $A \subset \tau_{2} \operatorname{cl}\left(\tau_{1}\right.$ int $\left.A\right)$. Again if $G$ is any $\tau_{1}$ open set satisfying $G \subset A \subset \tau_{2}$ clG, then $G \subset \tau_{1}$ int $A$. Hence $\tau_{1}$ int $A$ is the maximal $\tau_{1}$ open set contained in $A$ such that $\tau_{1}$ int $A \subset A \subset \tau_{2} \operatorname{cl}\left(\tau_{1}\right.$ int $\left.A\right)$. Taking $O=\tau_{1}$ int $A$, we get $\mathrm{O} \subset \mathrm{A} \subset \tau_{2} \mathrm{clO}$.

Conversely let $A \in \tau_{1}$ S.O. $(X)_{\tau_{2}}$. By the condition, there exists a maximal $\tau_{1}$ open set $\mathrm{O}$ such that $\mathrm{O} \subset \mathrm{A} \subset \tau_{2} \mathrm{clO} \ldots . . .(1)$.

If possible let $O \neq \tau_{1}$ int $A$. Then there exists a $\tau_{1}$ open set $G \subset A$ such that $G$ is not contained in $O$. Since $O \cup G$ is $\tau_{1}$ open set and $O \cup G \subset A \subset \tau_{2} \operatorname{clO} \subset \tau_{2} \operatorname{cl}(O \cup G)$, this contradicts that $O$ is maximal satisfying the condition (1). Hence $\tau_{1}$ int $A=O$ and so $\tau_{1}$ int $A$ is a $\tau_{1}$ open set, i.e., $\tau_{1} \operatorname{int} A \in \tau_{1}$. Therefore $\tau_{1} \operatorname{int}\left(\tau_{1}\right.$ S.O. $\left.(X)_{\tau_{2}}\right) \subset \tau_{1}$ and consequently $\tau_{1}=\tau_{1} \operatorname{int}\left(\tau_{1}\right.$ S.O. $\left.(X)_{\tau_{2}}\right)$.

Remark 3.18. We see that there is a bispace which is not bitopological space where the condition $\mathrm{C}_{2}$ holds. For, consider the bispace $\left(\mathrm{X}, \tau_{1}, \tau_{2}\right)$ as in Example 3.11 where the $\tau_{1}$ open sets are the only $\tau_{1}$ s.o.sets w.r.to $\tau_{2}$. So for any set $\mathrm{A}$ which $\tau_{1}$ s.o.w.r.to $\tau_{2}$ in $\mathrm{X}$, there exists a maximal $\tau_{1}$ open set $\mathrm{O}(=\mathrm{A})$ such that $\mathrm{O} \subset \mathrm{A} \subset \tau_{2} \mathrm{clO}$.

We now give a necessary and sufficient condition in terms of semi open sets for a bispace to be a bitopological space.

Theorem 3.19. A bispace $\left(\mathrm{X}, \tau_{1}, \tau_{2}\right)$ is a bitopological space if and only if following condition holds:

(i) arbitrary union of $\tau_{1}\left(\tau_{2}\right)$ s.o.sets w.r.to $\tau_{2}\left(\tau_{1}\right)$ is $\tau_{1}\left(\tau_{2}\right)$ s.o.w.r.to $\tau_{2}\left(\tau_{1}\right)$

(ii) $\tau_{1}=\tau_{1} \operatorname{int}\left(\tau_{1}\right.$ S.O. $\left.(X)_{\tau_{2}}\right)$ and $\tau_{2}=\tau_{2} \operatorname{int}\left(\tau_{2}\right.$ S.O. $\left.(X)_{\tau_{1}}\right)$.

Proof. If $\left(X, \tau_{1}, \tau_{2}\right)$ is a bitopological space then (i) holds $[3]$. For (ii), let $O \in \tau_{1}$. Then $O \in$ $\tau_{1}$ S.O. $(X)_{\tau_{2}}$ and since $O=\tau_{1} \operatorname{int} O, O \in \tau_{1} \operatorname{int}\left(\tau_{1}\right.$ S.O. $\left.(X)_{\tau_{2}}\right)$. Therefore, $\tau_{1} \subset \tau_{1} \operatorname{int}\left(\tau_{1}\right.$ S.O. $\left.(X)_{\tau_{2}}\right)$. On the other hand, let $O \in \tau_{1} \operatorname{int}\left(\tau_{1}\right.$ S.O. $\left.(X)_{\tau_{2}}\right)$. Then $O=\tau_{1}$ int $A$ for some $A \in \tau_{1}$ S.O. $(X)_{\tau_{2}}$ and hence $O \in \tau_{1}$. Therefore, $\tau_{1} \operatorname{int}\left(\tau_{1}\right.$ S.O. $\left.(X)_{\tau_{2}}\right) \subset \tau_{1}$. Therefore, $\tau_{1}=\tau_{1} \operatorname{int}\left(\tau_{1}\right.$ S.O. $\left.(X)_{\tau_{2}}\right)$. Similarly we can prove that $\tau_{2}=\tau_{2} \operatorname{int}\left(\tau_{2}\right.$ S.O. $\left.(X)_{\tau_{1}}\right)$.

Conversely, it suffices to show that an arbitrary union of $\tau_{1}\left(\tau_{2}\right)$ open sets is $\tau_{1}\left(\tau_{2}\right)$ open in 
$\left(X, \tau_{1}, \tau_{2}\right)$. Let $\left\{G_{i}\right\}$ be an arbitrary collection of $\tau_{1}$ open sets and $G=\cup G_{i}$. Each $G_{i}$ being $\tau_{1}$ open is $\tau_{1}$ s.o.w.r.to $\tau_{2}$. So by (i) $G$ is $\tau_{1}$ s.o.w.r.to $\tau_{2}$. Then by (ii) $\tau_{1}$ int $G$ is $\tau_{1}$ open. So $\tau_{1}$ int $G=G$ and similarly arbitrary union of $\tau_{2}$ open sets is $\tau_{2}$ open set and this proves the theorem.

Definition 3.20. ( $f f .[7])$ : Let $\left(\mathrm{X}, \tau_{1}, \tau_{2}\right)$ be a bispace. Two non empty subsets $\mathrm{A}$ and $\mathrm{B}$ are said to be

(i) pairwise weakly separated if there exist a $\tau_{1}$ open set $\mathrm{U}$ and $a \tau_{2}$ open set $\mathrm{V}$ such that $\mathrm{A} \subset \mathrm{U}$, $\mathrm{B} \subset \mathrm{V}, \mathrm{A} \cap \mathrm{V}=\phi, \mathrm{B} \cap \mathrm{U}=\phi$.

(ii) pairwise strongly separated if there exists a $\tau_{1}$ open set $\mathrm{U}$ and $a \tau_{2}$ open set $\mathrm{V}$ such that $\mathrm{A} \subset \mathrm{U}$, $\mathrm{B} \subset \mathrm{V}, \mathrm{U} \cap \mathrm{V}=\phi$.

Definition 3.21. (cf.[7] ): A subset $\mathrm{A}$ in a bispace $\left(\mathrm{X}, \tau_{1}, \tau_{2}\right)$ is said to be pairwise connected if it can not be expressed as the unions of two pairwise weakly separated sets.

Remark 3.22. In [3] it is proved that in a bitopological space $\left(\mathrm{X}, \tau_{1}, \tau_{2}\right)$ if $\mathrm{A}=\mathrm{O} \cup \mathrm{B}$ where (i) $\mathrm{O} \neq \phi$ is $\tau_{1}$ open (ii) $\mathrm{A}$ is pairwise connected and (iii) $\mathrm{B}^{\prime} \tau_{1}$, the derived set of $\mathrm{B}$ w.r.to $\tau_{1}$ is empty, then $\mathrm{A}$ is $\tau_{1}$ s.o.w.r.to $\tau_{2}$. But this is not true in a bispace as shown in the following example.

Example 3.23. Let $\mathrm{X}=([0,1]-\mathrm{Q}) \cup\{\sqrt{2}\}$ and $\left\{\mathrm{G}_{i}\right\}$ be the collection of all countable subsets of $[0,1]-Q$ where $Q$ is the set of all rational numbers. Let $\tau_{1}$ be the collection of all sets $G_{i} \cup\{\sqrt{2}\}$ together with $\mathrm{X}$ and $\phi$, and $\tau_{2}$ be the collection of all sets $\mathrm{G}_{i}$ together with $\mathrm{X}$ and $\phi$ where $\mathrm{G}_{i} \in\left\{\mathrm{G}_{i}\right\}$. Then $\left(X, \tau_{1}, \tau_{2}\right)$ is a bispace. Let $\mathrm{A}=\left(\left[0, \frac{1}{2}\right]-\mathrm{Q}\right) \cup\{\sqrt{2}\}$. Then $\mathrm{A}$ is pairwise connected, because if $A=A_{1} \cup A_{2}$, then at least one of $A_{1}$ and $A_{2}$ say $A_{1}$ is uncountable and $X$ is the only $\tau_{1}$ open set containing $A_{1}$. Let $\mathrm{G}$ be a nonempty countable subset of $\left[0, \frac{1}{2}\right]-\mathrm{Q}$ and $\mathrm{O}=\mathrm{G} \cup\{\sqrt{2}\}$. Then $\mathrm{A}=\mathrm{O} \cup(\mathrm{A}-\mathrm{O})$ where $\mathrm{O} \neq \phi$, is $\tau_{1}$ open. Also $(\mathrm{A}-\mathrm{O})^{\prime} \tau_{1}$, the set of all $\tau_{1}$ limit points of $\mathrm{A}-\mathrm{O}$ is empty. Indeed if $\alpha \in[0,1]-\mathrm{Q}$, then $\{\alpha, \sqrt{2}\}$ is a $\tau_{1}$ open set containing $\alpha$ satisfying $\{\alpha, \sqrt{2}\} \cap((A-O)-\{\alpha\})=\phi$. Again if $\alpha=\sqrt{2}$ then for any $p \in G,\{p, \alpha\}$ is a $\tau_{1}$ open set containing $\alpha$ satisfying $\{p, \alpha\} \cap((A-O)-\{\alpha\})=\phi$. Thus no point of $X$ can be a $\tau_{1}$ limit point of $\mathrm{A}-\mathrm{O}$.

So all the conditions stated in the Remark 3.22 above are satisfied, but $\mathrm{A}$ is not $\tau_{1}$ s.o.w.r.to $\tau_{2}$, because if $G_{i} \cup\{\sqrt{2}\}$ is any $\tau_{1}$ open set contained in $A$, then $\tau_{2} \operatorname{cl}\left(G_{i} \cup\{\sqrt{2}\}\right)=G_{i} \cup\{\sqrt{2}\}$.

However the following theorem is true.

Theorem 3.24. Let $\left(\mathrm{X}, \tau_{1}, \tau_{2}\right)$ be a bispace. If $\mathrm{A}=\mathrm{O} \cup \mathrm{B}$ where (i) $\mathrm{O}(\neq \phi)$ is $\tau_{1}$ open (ii) $\mathrm{A}$ is pairwise connected (iii) there exists a $\tau_{2}$ closed set $\mathrm{F}_{1} \supset \mathrm{O}$ such that $\mathrm{B} \cap \mathrm{F}_{1} \subset \mathrm{G} \subset \tau_{2}$ clO for some $\tau_{1}$ open set $\mathrm{G}$, then $\mathrm{A}$ is $\tau_{1}$ s.o.w.r.to $\tau_{2}$.

Proof. We show that $B \subset \tau_{2}$ clO. If $B \not \subset \tau_{2}$ clO then there exists a $\tau_{2}$ closed set $F \supset O$ such that $\mathrm{B} \not \subset \mathrm{F}$. Let $\mathrm{B}_{1}=\mathrm{B} \cap \mathrm{F}, \mathrm{B}_{2}=\mathrm{B}-\mathrm{B}_{1}$. Then $\mathrm{B}_{2} \subset X-\mathrm{F}$ and $\mathrm{B}_{2} \neq \phi$. Further, let $B_{1}^{*}=B_{1} \cap F_{1}$ and $B_{1}^{* *}=B_{1} \cap\left(X-F_{1}\right)$. Then $A=O \cup B=O \cup B_{1} \cup B_{2}=\left(O \cup B_{1}^{*}\right) \cup\left(B_{1}^{* *} \cup B_{2}\right)$. Now $B_{1}^{* *} \cup B_{2} \subset\left(X-F_{1}\right) \cup(X-F)=X-\left(F \cap F_{1}\right)=G_{2}$ (say), which is $\tau_{2}$ open. Since $B_{1}^{*}=$ 
$\mathrm{B}_{1} \cap \mathrm{F}_{1}=\mathrm{B} \cap \mathrm{F} \cap \mathrm{F}_{1} \subset \mathrm{B} \cap \mathrm{F}_{1} \subset \mathrm{G}$, we have $\mathrm{O} \cup \mathrm{B}_{1}^{*} \subset \mathrm{O} \cup \mathrm{G}=\mathrm{G}_{1}$ (say), which is $\tau_{1}$ open set. Since $\mathrm{G} \subset \tau_{2} \mathrm{clO} \subset \mathrm{F} \cap \mathrm{F}_{1}$ and $\mathrm{G}_{1}=\mathrm{O} \cup \mathrm{G} \subset \mathrm{F} \cap \mathrm{F}_{1}$, it follows that $\mathrm{G}_{1} \cap \mathrm{G}_{2}=\phi$. This implies that $\mathrm{O} \cup \mathrm{B}_{1}^{*}$, $\mathrm{B}_{1}^{* *} \cup \mathrm{B}_{2}$ are non empty strongly separated sets, a contradiction. Hence $\mathrm{O} \subset \mathrm{A}=\mathrm{O} \cup \mathrm{B} \subset \tau_{2} \mathrm{clO}$ and so $A$ is $\tau_{1}$ s.o.w.r.to $\tau_{2}$.

Received: October 2013. Accepted: November 2014.

\section{References}

[1] Alexandroff A.D., Additive set functions in abstract spaces, (a) Mat.Sb.(N.S), 8:50(1940), 307348.(English,Russian Summary). (b) ibid, 9:51(1941), 563-628, (English,Russian Summary)

[2] Biswas N., On Semi Open Mapping in topological spaces, Bull.Cal.Math.Soc.(1969).

[3] Bose S., Semi open sets, Semi continuity and Semi Open mapping in bitopological spaces. Bull.Cal.Math.Soc. 73. 237-246 (1981).

[4] Das P. and Rashid M.A., $\mathrm{g}^{*}$-closed sets and a new separation axiom in Alexandroff spaces,Arch. Math. (Brno),30(2003),299-307.

[5] Das P. and Rashid M.A., Semi $\mathrm{g}^{*}$-closed sets and a new separation axiom in the spaces, Bulletine of the Allahabad Mathematical Society, Vol. 19, (2004), 87-98.

[6] Kelley J.C., Bitopological spaces, Proc.London.Math.Soc. 13,71(1963).

[7] Lahiri B.K. and Das P., Semi Open set in a space, Sains malaysiana 24(4) 1-11(1995).

[8] Lahiri B.K. and Das P., Certain bitopological concept in a Bispace, Soochow J.of Math.Vol.27.No.2,pp.175-185(2001).

[9] Levine N., Semi Open sets and Semi Continuity in topological spaces, Amer.Math.Monthly $70,36(1963)$.

[10] Noiri Takashi, Remarks on Semi Open Mapping, Bull.Cal.Math.Soc.65,197(1973).

[11] Pervin W.J., Connectedness in Bitopological spaces, Ind.Math.29,369(1967).

[12] Reilly I.L., On Bitopological separation Properties, Nanta Math.5,14-25(1972).

[13] Sikorski R., Closure Homomorphism and Interior Mapping, Fund.Math 41,12-20(1995). 